\title{
Palabras pronunciadas por la Porrrectora de la Universidad de Chile, profesora Rosa Devés, con motivo de la celebración de los 10 años del Centro Heidelberg
}

Es un honor para la Universidad de Chile participar en la celebración de los 10 años del Heidelberg Center para América Latina. Por una década este espacio de diálogo académico nos ha enriquecido como institución y -aún más importante- nos ha permitido cooperar en la formación de graduados y profesionales en un espacio académico internacionalizado, diverso y, por lo tanto, más fértil para la generación de ideas y la comprensión de procesos complejos.

Quienes estamos aquí hoy, entendemos la importancia de construir desde el conocimiento, especialmente desde aquel conocimiento que somos capaces de crear en conjunto, en un proceso atento a las necesidades y expectativas de los otros. Así es como hemos colaborado en campos como el derecho, la psicoterapia, la informática médica y las geociencias. Esta selección de áreas ha resultado de considerar las intersecciones de las capacidades mutuas y la relevancia de los problemas que surgen en estos contextos, siempre cuidando de mantener el delicado balance entre las capacidades existentes y la posibilidad de desarrollar algo nuevo.

Pero el que esta importante reunión de la educación, la ciencia y la política alemana y chilena se realice en estos días conlleva para la Universidad de Chile un simbolismo adicional, porque -precisamente ahora- estamos trabajando con mucha 
intensidad en un proyecto para instalar a la Educación como un área disciplinaria de importancia mayor en la Universidad, lo que no ha sido el caso desde la separación del Instituto Pedagógico por la dictadura en 1981.

El cambio que proyectamos requiere involucrar a la Universidad en un proceso de innovación, para generar nuevos programas y generar cambios sistémicos que permitan asociar efectiva y estrechamente la formación, la investigación y la vinculación con el medio en educación. Los nuevos programas estarán abiertos a aprender de: i) la realidad nacional, a través de su interacción con las escuelas y sus comunidades; ii) de la investigación en general, incluida aquella sobre los propios programas; iii) de las mejores experiencias internacionales, mediante el trabajo conjunto; y iv) de la cooperación con otras universidades chilenas, especialmente las estatales.

Por ello, y porque el futuro debe construirse a partir de la historia, hemos estado analizando desde la perspectiva estratégica aquellos procesos a través de los cuales la reforma alemana impactó tan fuertemente la educación chilena, especialmente la educación secundaria, pero también la educación normal, a través de un proceso de transferencia propiciado desde el Estado. Este propósito tuvo como su máxima expresión la creación del Instituto Pedagógico en 1889, la primera institución formadora de docentes secundarios en el país y por 30 años, la única. Fue así como un grupo de ocho profesores y científicos alemanes se hicieron cargo de las primeras actividades docentes y del diseño del primer plan de estudios. El cerebro y motor de este proceso fundacional fue Valentín Letelier, quien en su estadía en Berlin entre 1881 y 1885 había estudiado en profundidad el sistema educacional alemán en sus diversas dimensiones desde la administración escolar hasta los métodos didácticos.

Así en Chile, el discurso del filósofo alemán Johann Friedrich Herbart -que entendía a la pedagogía como una cienciay los conceptos de la Universidad humboldtiana, confluyeron para dar origen a una preparación de profesores que se basó en una formación disciplinar rigurosa y especializada, y en la producción de conocimiento, no solo en su transmisión. Esto se vio reflejado en las prácticas experimentales de la pedagogía y la psicología dentro del Instituto, y en su valoración del 
método inductivo, a partir de la observación directa de las cosas y de la experimentación en la enseñanza.

Estos principios encuentran gran resonancia en la aspiraciones actuales de la Universidad de Chile para la formación pedagógica.

Aprovechamos este momento tan especial para invitarlos a acompañarnos en este desarrollo. No se trata aquí de exportar o importar productos educativos, porque cuando hablamos de educación estamos hablando de procesos complejos, de visiones de mundo y de transformaciones que con seguridad representarán aprendizajes para todos. Se trata ni más ni menos que de construir juntos a partir de la reflexión, la investigación y procesos sociales vivos un futuro para nuestros niños.

Por ello, 120 años más tarde estamos comprometidos a continuar trabajando para conseguir ese resultado que Valentín Letelier describe en su informe sobre «Las Escuelas de Berlín»: "La enseñanza misma se hace con tal arte que las clases más parecen de placer que de trabajo. Especialmente la de silabario, que en las de Chile es la más fatigosa, en Ias de Berlin cautiva toda la atención de los niños, y los agita, y despierta en ellos la emulación y el deseo de saber y prosperar. Al ver la animación de algunas clases de silabario, uno llega a imaginarse estar presenciando un certamen de alegría». 